Itinéraires Itinéraires

Littérature, textes, cultures

\title{
La vie d'Ahmed/Zahra ou la mise en crise de la masculinité chez Tahar Ben Jelloun
}

Yves Clavaron

\section{(2) OpenEdition}

1 Journals

Édition électronique

URL : https://journals.openedition.org/itineraires/2219

DOI : 10.4000/itineraires.2219

ISSN : 2427-920X

Éditeur

Pléiade

Édition imprimée

Date de publication : 1 décembre 2008

Pagination : 149-161

ISBN : 978-2-296-07519-1

ISSN : 2100-1340

\section{Référence électronique}

Yves Clavaron, «La vie d'Ahmed/Zahra ou la mise en crise de la masculinité chez Tahar Ben Jelloun », Itinéraires [En ligne], Numéro inaugural | 2008, mis en ligne le 01 décembre 2008, consulté le 21 septembre 2021. URL : http://journals.openedition.org/itineraires/2219 ; DOI : https://doi.org/ 10.4000/itineraires.2219

\section{(c) (i) () $\Theta$}

Itinéraires est mis à disposition selon les termes de la licence Creative Commons Attribution - Pas d'Utilisation Commerciale - Pas de Modification 4.0 International. 


\title{
La vie d'Ahmed/Zahra ou la mise en crise de la masculinité chez Tahar Ben Jelloun
}

\begin{abstract}
L'Enfant de sable and La Nuit sacrée form the diptych which made Tahar Ben Jelloun famous. L'Enfant de sable tells the story of a girl brought up as a boy called Ahmed in a male chauvinist society where she usurps men's rights and powers. In La Nuit sacrée, Ahmed comes back to life as Zahra, an old woman who tells the (true?) story of her painful path to recover her womanhood and reach a doubtful liberation. Drawing on the works of Judith Butler, Thomas Laqueur and Élisabeth Badinter, this article examines the ways in which Ben Jelloun tackles the question of the construction of masculinity through the example of this girl compelled to be male, and how the very form of fiction (genre, narrative structures, registers...) is informed by the construction of gender.
\end{abstract}

L'Enfant de sable et La Nuit sacrée forment le diptyque qui a rendu célèbre Tahar Ben Jelloun ${ }^{1}$. Il y met en scène une société arabo-musulmane, traditionnelle et figée, obéissant à un ordre ancestral et à une conception archaïque de la masculinité, hérités de la Jahilia, la période anté-islamique. Sans la nommer explicitement, les deux romans font aussi allusion à la charia, la loi islamique, qui consacre un système patriarcal et hiérarchisé, réservant tous les droits au chef de famille et réduisant la femme au statut d'un objet docile et soumis ${ }^{2}$. Ben Jelloun considère néanmoins que ces principes archaïques de masculinité sont alimentés par une lecture masculine du Coran, qui interprète le texte sacré dans le sens de ses intérêts.

1. T. Ben Jelloun, L'Enfant de sable, Paris, Le Seuil, coll. « Points Romans », 1985 et La Nuit sacrée, Le Seuil, coll. «Points Romans », 1987. Les références seront respectivement précédées des mentions ES et NS.

2. J. Minces, Le Coran et les Femmes, Paris, Hachette, 1996, p. 78-79. 
Le point de départ de L'Enfant de sable repose bien sur une question de masculinité: ne pas avoir d'enfant de sexe mâle vaut comme signe d'impuissance pour un homme musulman. Même s'il rejette la faute sur le ventre de sa femme qui a fait « fille sur fille jusqu'à la haine du corps » (ES, p. 19), Hadj Souleimane veut prouver sa virilité à la société. Pour cela, il décrète qu'après sept filles, le/la huitième né(e) s'appellera Ahmed pour mettre fin à la fatalité qui le poursuit. L'histoire de L'Enfant de sable est donc celle d'une fille élevée en garçon dans une société phallocratique, où elle usurpe les droits et privilèges des hommes. Ce roman d'éducation particulier se termine par une apocalypse : disparition tragique de tout l'entourage d'Ahmed, expulsion du conteur de la place « sous l'instigation de jeunes urbanistes technocrates » (ES, p. 135), perte du manuscrit et dislocation du texte en une série de variantes. Quelle que soit la version narrative, l'avenir de cet homme-femme reste chaotique et problématique, finissant à la « porte des sables » (ES, p. 199), la porte du désert. La suite, La Nuit sacrée, fait renaître Ahmed sous les traits de Zahra, une vieille femme qui fait le (vrai?) récit de son douloureux itinéraire pour réintégrer sa féminité et obtenir une libération à laquelle elle ne parvient jamais totalement.

Parler d'une crise de la masculinité est devenu un lieu commun, dont Élisabeth Badinter dresse un historique à partir de la culture occidentale et, si elle puise de nombreux exemples chez des peuples d'Asie, d'Afrique ou d'Océanie, elle n'évoque pas les sociétés arabo-musulmanes, que Ben Jelloun met en scène ${ }^{3}$. En revanche, Pierre Bourdieu étudie l'inconscient androcentrique de la société berbère de Kabylie et montre comment 1'opposition des genres est informée par des mythes cosmogoniques qui conditionnent symboliquement les femmes à une véritable incorporation de la domination masculine ${ }^{4}$.

À travers l'histoire de cette enfant sommée d'être un homme, Ben Jelloun pose la question de la construction du genre masculin, et d'une manière qui peut se lire, entre autres, au filtre des théories de la performativité du genre de Judith Butler ${ }^{5}$. Mais construction du genre et élaboration de la fiction vont de pair, et l'évolution d'un roman à l'autre semble réduire l'audace de l'innovation formelle et ramener la problématique du genre (gender) à des schémas traditionnels.

3. É. Badinter, XY: de l'identité masculine, Paris, Odile Jacob, 1992, p. 25.

4. P. Bourdieu, La Domination masculine, Paris, Le Seuil, 1998, p. 28.

5. « Le genre se révèle performatif - c'est-à-dire qu'il constitue l'être qu'il est censé être. Ainsi le genre est toujours un faire, mais non le fait d'un sujet qui précéderait ce faire », J. Butler, Trouble dans le genre: pour un féminisme de la subversion, trad. C. Kraus, Paris, La Découverte, 2005, p. 96; Gender Trouble: Feminism and the Politics of Subversion (1990), Londres et New York, Routledge, 1999. 


\section{La construction de la masculinité ou la performativité du genre}

On connaît la précarité de la distinction analytique entre sexe et genre: le premier relève d'une catégorie biologique tandis que le second serait produit par des processus sociaux et culturels. L'étude historique de Thomas Laqueur marque bien l'instabilité de ces notions. En effet, avant le XVIII ${ }^{\mathrm{e}}$ siècle, le sexe était considéré comme un épiphénomène, alors que le genre, considéré aujourd'hui comme une catégorie culturelle, était premier: être un homme ou une femme était avant tout un rang, une place dans la société ${ }^{6}$. La situation se complique encore si l'on ajoute la catégorie de la sexualité, qui relève d'une construction individuelle du sujet et ne dépend pas nécessairement du sexe. Le discours scientifique lui-même a été sexué de façon dichotomique et selon les oppositions traditionnelles de genre, car connaissances biologiques et pratiques sociales vont de pair comme le montrent un certain nombre de féministes? ${ }^{7}$ Interprétant l'« être » du genre comme un effet, Butler le soumet à une enquête visant à « esquisser les paramètres politiques de sa construction sur le mode de l'ontologie ${ }^{8} \gg$.

La société mise en scène par Ben Jelloun dans ses deux romans est hétéronormative, c'est-à-dire qu'elle institue un système asymétrique et binaire de genre, qui tolère deux sexes et deux seulement, où le genre concorde parfaitement avec le sexe et où l'hétérosexualité (reproductive) est obligatoire, en tout cas désirable et convenable. La binarité du système renvoie à un ordre social profondément inégalitaire - « Dans ce pays, tu réprimes ou tu es réprimé » (ES, p. 121) - et à une structure familiale hiérarchisée opposant «le père tout-puissant » et «les femmes reléguées à la domesticité avec une parcelle d'autorité que leur laisse le mâle » (ES, p. 89).

\section{La performativité du genre}

C'est donc dans une société où le genre et le sexe connaissent une assignation stricte que Ben Jelloun décrit le passage transgénérique d'une fille non désirée à la naissance. Et ce sont la volonté et la parole paternelles qui instituent ce changement dans un texte qui se place d'ailleurs tout entier sous le signe de la parole et de la performance orale. Après un rêve où lui apparaît la mort sous les traits d'un adolescent androgyne, le père décrète: "L'enfant à naître sera un mâle, même si c'est une fille! 》 (ES, p. 21), parole qui est ensuite transmise à la mère: «L'enfant que tu mettras au monde sera un mâle, ce sera un homme, il s'appellera Ahmed même si c'est une fille! »; "Il sera élevé dans la tradition réservée aux mâles »

6. T. Laqueur, La Fabrique du sexe: essai sur le corps et le genre en Occident, trad. M. Gautier, Paris, Gallimard, coll. « NRF Essais », 1992.

7. Voir É. Peyre et J. Wiels, « Le sexe biologique et sa relation au sexe social », Les Temps modernes, $\mathrm{n}^{\circ}$ 593, 1997, p. 14-48.

8. Butler, Trouble dans le genre, p. 109. 
(ES, p. 23). À la naissance, contre toute évidence biologique, la parole performative du père (et de la sage-femme) crée l'être masculin: "C'est un homme, un homme, un homme. » (ES, p. 26) Le texte met en scène le processus décrit par Butler comme « boying », par opposition à « girling », ce qui fait du genre le résultat pragmatique d'un énoncé'.

À propos du drag queen, Butler parle de performance inversée du genre, mais précise que le genre ne se réduit pas à une action théâtrale: il ne suffit pas de se travestir pour subvertir la puissance du genre. Pour elle, l'action du genre requiert une performance répétée: elle est réitération tout comme elle est assignation normative. Le genre n'est pas notre essence, qui se révélerait dans nos pratiques, mais ce sont les pratiques du corps dont la répétition institue le genre: "L'idée que le genre est performatif a été conçue pour montrer que ce que nous voyons dans le genre comme une essence intérieure est fabriqué à travers une série ininterrompue d'actes, que cette essence est posée en tant que telle dans et par la stylisation genrée du corps ${ }^{10}$. \ Ce que signifie Butler, c'est que l'identité sexuelle ne préexiste pas à nos actions car celles-ci sont elles-mêmes agies - conditionnées: le genre est l'effet des normes de genre.

Cette allégation se vérifie dans la construction de la masculinité d'Ahmed. Le père, démiurge plus que géniteur, façonne un corps masculin par son discours: «Regarde comme il est beau, touche ses petits testicules, touche son pénis, c'est déjà un homme. » (ES, p. 27) Dès lors, il grandira « selon la loi du père » (ES, p. 32). Badinter dit nettement que la masculinité est une réaction plus qu'une adhésion, une protestation de virilité pour effacer tout soupçon de féminité ${ }^{11}$. La régulation du genre s'exprime donc en termes de restriction, de prohibition et de régulation. Battu par d'autres enfants, Ahmed reçoit une gifle: « tu n'es pas une fille pour pleurer! Un homme ne pleure pas. » (ES, p. 39) La petite fille devient un mâle par une série de pratiques sociales dictées par le père : la coercition du corps (poitrine bandée, cheveux coupés tous les mois et interdiction du henné « réservé aux filles »), une parodie de circoncision et une éducation à l'école coranique avec ses pairs, «d'autres garçons » (ES, p. 32-33). Au hammam, il quitte un jour le côté des femmes, espace de la parole et des mots interdits «autour du sexe » (ES, p. 35), pour celui des hommes, communauté du silence, des plaisirs illicites et des épanchements solitaires. C'est d'ailleurs au hammam, espace où les corps se mettent à nu, que se déroule une scène très lacanienne: Ahmed regarde son bas-ventre dans un miroir afin de se convaincre que le sien ne ressemble pas à ceux "charnus et poilus » des femmes qu'il côtoie au bain (ES, p. 36). La construction de la masculinité

9. J. Butler, Bodies that Matter: On the Discursive Limits of « Sex », Londres et New York, Routledge, 1997, p. 7.

10. Butler, Trouble dans le genre, introduction de 1999, p. 36.

11. Badinter, op. cit., p. 92. 
passe par une série d'épreuves qui requiert un certain stoïcisme de la part du jeune homme. Cependant, si Ahmed se construit en homme par l'injonction paternelle, il devient aussi féminin par l'expérience (passive) de la menstruation (ES, p. 46). À l'image de ces créatures qui finissent par dépasser leur créateur, Ahmed veut prendre le jeu à son compte et substituer sa propre volonté à celle de son père: "Père, tu m'as fait homme, je dois le rester. » (ES, p. 51) Radicalisant les normes de genre qu'il énonce - la femme se soumet et s'enveloppe dans « un linceul de silence » (ES, p. 53) -, il décide de confronter sa masculinité à une épreuve suprême: le mariage avec sa cousine Fatima, fille réprouvée car épileptique. Le « oui » du mariage hétérosexuel est le second performatif décisif après celui de la naissance, selon Butler, or, là encore, il y a détournement du genre.

Dans La Nuit sacrée, Ahmed fait le chemin à l'envers pour devenir Zahra. Néanmoins, il a déjà entamé ce parcours dans L'Enfant de sable et le retour passe par une " performance » théâtrale puisque dans une fête foraine, à côté de la « femme à barbe », on lui propose de jouer un rôle androgyne-homme puis femme fatale (ES, p. 121). S'affirme ici la dimension parodique qu'évoque Butler à propos du drag queen chez celui qui sera "l'homme aux seins de femme ». Mais cette posture «ne présuppose pas l'existence d'un original qui serait imité », l'imitation serait « sans original ${ }^{12}$. Pour Butler, il s'agit de prendre appui sur les exceptions (comme le drag queen) pour penser la règle, non comme inversion, mais comme modèle d'invention de nouvelles formations du sujet et, en même temps, comme vérité générale de la norme. C'est l'exception, l'étrange qui permet de comprendre comment est constitué le monde ordinaire, que l'on considère comme "normal », des significations sexuelles. Le jeu du travesti, parodique, fait prendre conscience de ce que l'on joue un rôle, quel que soit son propre degré de conformité aux normes de genre et de sexualité. Même si le protagoniste, Ahmed/Zahra, ne va pas jusqu'aux performances ostentatoires du travesti, on observe chez elle/lui tout un jeu sur les composantes de son identité corporelle: le sexe anatomique, l'identité de genre et la performance du genre.

« Redevenue femme, du moins reconnue comme telle par le géniteur » (NS, p. 34), Zahra peut tenter de mettre fin à vingt ans de mensonge, car elle a été libérée par la parole du père mourant. Avant de mourir, ce dernier résume son « œuvre » : " Que de fois, je dus te rappeler que tu étais un petit homme, un garçon », avant de décréter: «Tu viens de naître... Tu es une femme. » (NS, p. 32) La construction du genre apparaît bien comme une performance: «Redevenue femme [...], j'avais encore à jouer le jeu, le temps de régler les affaires de succession et d'héritage. » (NS, p. 34) Elle doit ensuite plier son corps à de nouvelles postures, découvrir un nouveau langage corporel: «marcher avec souplesse» (NS, p. 35) ou se déplacer 
dans la rue «en tenant un homme par le bras » (NS, p. 123). Toutefois, pour avoir trop longtemps vécu dans le simulacre, Zahra reste prisonnière de son histoire et fixée sur le « monstre » que son père avait fabriqué (NS, p. 55 et 172). Ce blocage amplifie son aliénation: « Mon corps s'était arrêté dans son évolution; il ne muait plus $[. .$.$] ni un corps de femme plein$ et avide, ni un corps d'homme serein et fort; j'étais entre les deux, c'est-àdire en enfer. » (NS, p. 178) N'ayant pas pris un genre fixe, Ahmed/Zahra s'avère inintelligible pour les autres mais aussi à lui/elle-même.

Pour Butler, la masculinité n'est pas une essence mais une idéologie, qui tend à justifier la domination masculine. Le corps existe, mais il est le produit d'une histoire sociale incorporée. Néanmoins, l'emprise des normes n'empêche pas de penser une prise sur les normes et d'avoir une réflexion sur celles-ci. Et, de fait, le personnage Ahmed/Zahra présente une conscience lucide de l'impact des forces sociales et politiques dans la fabrication du genre: il/elle dit la force de la loi patriarcale qui pèse sur les êtres dans la société arabo-musulmane, notamment la subordination sexualisée de la femme et la violence qui pèse sur elle du fait de l'ordre phallocentrique. Butler concède que l'on ne peut parler d'un pur déterminisme car la matérialité du corps possède ses propres lois et obéit à une nature, mais elle choisit de s'intéresser au corps, non comme réalité préalable, mais comme effet bien réel des régulations sociales et des assignations normatives ${ }^{13}$.

\section{Le pouvoir, l'errance et la construction du genre}

En effet, pour Joan W. Scott, le genre est moins la construction sociale de la différence des sexes qu' « une façon première de signifier des rapports de pouvoir » et ces derniers s'incarnent dans la société patriarcale qui tente d'assujettir et de contenir la femme ${ }^{14}$. Comme pour échapper à l'action du pouvoir sur la construction du genre, les personnages de Ben Jelloun deviennent mobiles dans l'espace et la structure des romans s'organise davantage selon la spatialité que selon la chronologie: « J'ai perdu la notion du temps », reconnaît Ahmed (ES, p. 105). Ainsi, la révolte du héros se traduit par sa décision de partir et consiste en la revendication d'un retour à son corps de femme, qu'il libère des bandages lui enserrant la poitrine et auquel il donne du plaisir par la caresse (ES, p. 111). De même, dans La Nuit sacrée, Ahmed/Zahra se lance dans un long périple qui doit lui permettre de défaire le genre qui lui a été imposé. L'errance géographique est associée aux variations du genre, à l'image d'Antar, « un homme exemplaire, au courage légendaire », qui est en fait une femme et

13. Voir Butler, Bodies That Matter.

14. J. W. Scott, « Genre: une catégorie utile d'analyse historique », « Le genre de l'histoire », Les Cahiers du GRIF, n 37-38, printemps 1988, p. 125-153; " Gender: A Useful Category of Historical Analysis ", dans Gender and the Politics of History, New York, Columbia University Press, 1988. 
est devenu, après sa mort, « un saint ou une sainte [...] le marabout de l'errance » (ES, p. 84-85).

Suivant les théories de Michel Foucault, Butler explique que nous sommes constitués en tant que sujets par le pouvoir ${ }^{15}$. «Il y a une plastie du langage sur le réel », écrit Monique Wittig, mais cette conception foucaldienne qui fait du langage une sorte de prison peut être contestée ${ }^{16}$. Penser les normes qui nous définissent amène à reformuler la question de la domination, pour la poser en termes de pouvoir. Cela revient à dire que le pouvoir ne réprime pas seulement, il fait exister. L'assignation que nous endossons et reprenons à notre compte est la condition paradoxale de notre capacité, voire de notre puissance d'agir (agency). Ahmed/Zahra est une femme faite homme par une volonté masculine, puis une femme qui souhaite renouer avec son identité corporelle, mais sans pour autant accepter les rôles sociaux impartis au genre féminin. En cela, elle expérimente toute l'asymétrie qui existe entre une masculinité se construisant de manière autonome, et une féminité s'organisant dans sa relation à l'autre sexe ${ }^{17}$. Par les modulations de son genre, Ahmed/Zahra entre bien dans le programme de Butler, pour laquelle l'exception et l'étrange constituent une propédeutique à la remise en cause des catégories toutes faites.

Si Ben Jelloun donne à voir le caractère véritablement transformationnel et performatif du genre, notamment masculin, ce qui lui permet de dépasser la logique binaire et hiérarchisante de la différence sexuelle, il n'en demeure pas moins que le discours romanesque n'échappe pas complètement au dualisme et vient fournir au système - l'hégémonie masculine et la structure patriarcale - les arguments de sa propre perpétuation.

\section{Jeux de la fiction ou le retour aux normes de genre}

La différence de structure narrative entre les deux romans, tout en soulignant l'impossible réconciliation du masculin et du féminin dans l'entreprise de libération amorcée par Ahmed/Zahra, signale finalement le retour en force de la Loi et des stéréotypes du genre, comme si la réalité de la transgression ne pouvait être maintenue. C'est un certain type de littérature qui prend alors le relais, le conte et aussi la poésie, reléguant la problématique du genre dans un univers fantastico-merveilleux.

\section{De la polyphonie à la monophonie}

La parole se met en scène sur la place Jemaa El Fna de Marrakech, véritable forum public, sinuant sous les volutes de l'hyperbole et les ara-

15. Butler, Trouble dans le genre, p. 60.

16. Citée par Butler, Trouble dans le genre, p. 230.

17. Voir I. Löwy, L'Emprise du genre: masculinité, féminité, inégalité, Paris, La Dispute, coll. « Le genre du monde », 2006, quatrième de couverture. 
besques de la métaphore, puis s'égarant dans le dédale des sept portes de la médina. Comme le montre Laurence Kohn-Pireaux, L'Enfant de sable est pris en charge par une chaîne de sept conteurs, chacun abandonnant son histoire pour céder la parole à un autre conteur ${ }^{18}$. Le premier, anonyme, transmet la parole à Si Abdel Malek, le frère de Fatima. Quand le manuscrit disparaît, les conteurs rêvent, chacun à sa manière, l'histoire d'Ahmed/Zahra, qu'il s'agisse de Salem, le fils d'un esclave ramené du Sénégal, d'Amar, le vieil instituteur retraité ou de Fatouma. Ensuite, le troubadour aveugle, en qui l'on reconnaît Borges, prétend avoir rencontré Ahmed, redevenu femme, à Buenos Aires, avant de laisser la parole à l'étranger au turban bleu, qui conclut, mais sans l'achever, l'histoire. Symboliquement, il renvoie le lecteur à un astre peu bavard pour connaître le dénouement la lune, « quand elle sera entièrement pleine » (ES, p. 209).

La construction puis la déconstruction de la masculinité d'Ahmed/ Zahra passent ainsi par une esthétique du fragment: «Fragmentaire mais non dépourvu de sens, l'événement s'impose à ma conscience de tous les côtés. Le manuscrit que je voulais lire tombe en morceaux [...]. » (ES, p. 108) Chaque fragment, chaque microrécit constitue un nouveau commencement narratif qui tente de pallier le manque dans lequel s'origine le texte, travaillé par son « inassouvissement narratif ${ }^{19}$ ». La parole publique du conteur dévoile l'écriture secrète du journal intime rédigé par Ahmed/ Zahra, dont le roman donne à lire quelques extraits, mais ce texte se caractérise par des lacunes, des ellipses - « un espace blanc, des pages nues » (ES, p. 41-42), où il est demandé au lecteur/auditeur d'imaginer l'adolescence du personnage. Faute de pouvoir être racontée jusqu'au bout - les conteurs multiplient les défaillances -, l'histoire s'interrompt, laissée à l'interprétation du public, incapable de dire le devenir d'un être au genre ambivalent, un véritable être de sable, liquide minéral impossible à modeler et à saisir ${ }^{20}$. La parole se livre à une incessante correction du récit, conjugue répétition et altérité, différence et différé et, en cela, procède de la déconstruction derridienne, d'une écriture de la différance. La genèse du sujet se fait dans l'espacement, l'écartement: le personnage d'Ahmed/Zahra existe « entre », il explore infiniment de nouveaux contextes, déploie des simulacres dans une interaction constante du féminin et du masculin, mais résiste à toute assignation à une identité fixe ${ }^{21}$. Le roman

18. L. Kohn-Pireaux, Tahar Ben Jelloun, "L'Enfant de sable » et "La Nuit sacrée », Paris, Ellipses, 2000, p. 18.

19. Voir l'étude de R. Elbaz, Tahar Ben Jelloun ou l'Inassouvissement du désir narratif, Paris, L'Harmattan, 1996, p. 38.

20. A. Hafez-Ergaut, «Jeux de masques: L'Enfant de sable et La Nuit sacrée de Tahar Ben Jelloun », <http://www.arts.uwa.edu.au/MotsPluriels/MP1099ahe.html> (consulté le $1^{\text {er }}$ septembre 2007).

21. Voir la lecture de J. Derrida faite par F. Regard, L'Écriture féminine en Angleterre, Paris, PUF, coll. « Perspectives anglo-saxonnes », 2002, p. 72-81. 
L'Enfant de sable élabore ainsi une véritable cryptographie, échouant à dire le secret de l'être.

La fragmentation extrême de la descente aux enfers que constitue L'Enfant de sable se résout dans La Nuit sacrée. Certes, l'on retrouve le conteur au turban bleu au début du roman, mais c'est Zahra qui assume son propre mandat narratif. Hormis quelques narrateurs secondaires ponctuels, La Nuit sacrée prend une allure autobiographique et met en scène une voix unifiante, celle d'Ahmed/Zahra devenu(e) femme, apparemment libéré(e) du rôle que son père lui avait assigné. La parole restituée dit l'histoire incorporée de/par Zahra: parole de témoignage, de souffrance et d'affirmation de soi, de celle/celui qui a voulu disposer de son corps et de sa liberté, malgré la marginalité et l'errance. À sa manière, ce personnage de femme marocaine semble contredire les allégations de Gayatri Spivak, pour laquelle il n'y a pas de lieu possible d'expression pour la femme subalterne ${ }^{22}$. En fait, le récit de Zahra, relayé par le consul qui tente de dénouer le labyrinthe de son histoire par l'écriture (NS, p. 169), retrace l'itinéraire d'un être qui s'efforce de reconstruire son genre, mais au prix d'un retour aux principes de la masculinité la plus archaïque. Parallèlement, de L'Enfant de sable à La Nuit sacrée, Ben Jelloun passe d'une esthétique postmoderniste à la Borges à une forme romanesque plus canonique, comme s'il voulait effacer les audaces de l'expérimentation mise en œuvre pour la construction « genrée » de son protagoniste.

\section{Le retour aux stéréotypes du genre féminin}

Libérée et re-née la vingt-septième nuit du Ramadan, celle de la première révélation faite à Mahomet par l'archange Gabriel dans une grotte du mont Hira en Arabie, Zahra accomplit un parcours qui, loin de la libérer, l'enferme dans le cycle des malheurs réservés aux femmes, dans la réification d'une configuration ancestrale qui hiérarchise les genres. Zahra, ou Ahmed dans sa vie antérieure, connaît une série d'épreuves dans sa quête d'identité et de féminité, imposée par la violence de la société patriarcale. Tout d'abord, c'est par un viol commis par un homme « sans visage » qu'elle devient femme (NS, p. 63). L'image évoquant cet acte est sans doute ambivalente, « $[\mathrm{u}] \mathrm{n}$ poignard caressant le dos » (NS, p. 58), mais elle conforte d'une certaine façon un préjugé masculin, selon lequel le viol est toujours secrètement désiré par la victime. Quant au premier acte sexuel consenti avec l'homme qu'elle aime, le consul, il s'accomplit dans le cadre du bordel et par ruse: Zahra se fait passer pour une prostituée, en profitant de ce que son partenaire est aveugle (NS, p. 126). Zahra se trouve plus tard condamnée à quinze ans de prison pour le meurtre de son oncle, le père de

22. "There is no space from which the sexed subaltern can speak », G. C. Spivak, «Can the Subaltern Speak? », dans P. Williams et L. Chrisman, dir., Colonial Discourse and Post-Colonial Theory: A Reader, Hemel Hempstead, Harvester Wheatsheaf, 1993, p. 103. 
Fatima, son épouse lorsqu'elle était Ahmed. Dans une scène aux limites du fantastique, ses sœurs reviennent, membres d'une « secte de sœurs musulmanes, fanatiques et brutales »(NS, p. 157), version arabo-islamique des Euménides ou des Érinyes. Avides de se venger de l'usurpation du supposé Ahmed, qui leur a indûment imposé une tutelle masculine, elles s'attaquent à la féminité même de Zahra: après l'avoir tondue, elles pratiquent une excision sauvage, lui cousent le sexe, contrepoint tragique du simulacre de circoncision qu'avait subi Ahmed enfant. La plupart des expériences de Zahra dans la reconquête de sa féminité tendent à illustrer l'aliénation du corps féminin, brutalement soumis à la loi d'une société phallocratique et rarement sujet de son désir propre.

Un destin tragique pèse sur Ahmed/Zahra, peut-être provoqué par l'hybris du père qui avait voulu façonner un homme à partir d'un corps féminin, et cette faute paternelle, endossée par le héros qui a pris le jeu à son compte et l'a radicalisé ${ }^{23}$, semble peser jusqu'au bout. Grâce à l'amour du consul, l'enfant de sable est certes devenu une « statue de chair désirée et désirante » (NS, p. 138), mais elle continue à considérer son corps comme « un sac de sable » (NS, p. 167). Malédiction et/ou disposition personnelle, Zahra est vouée à l'incomplétude et au malheur, qui revêt les formes multiples de la répression imposée aux femmes et illustre indirectement le déterminisme qui agit sur la construction du genre. Un graffiti représentant un « vagin avec des dents » (NS, p. 77), avatar du vagin denté de « Bérénice » d'E. A. Poe, allégorise la difficulté d'être femme. En définitive, la libération du désir passe pour Zahra par un champ de ruines et de morts - maison effondrée du père, assassinat de l'oncle, violences de la prison -, et le parcours initiatique qui la malmène - on serait tenté de dire « mâle-mène »-ne remet pas en cause la hiérarchie des genres édifiée par la société arabo-musulmane. Le désir provoque certes un élan et une régénération, mais l'être féminin n'est pas décolonisé. Toutefois, ce dernier accède à la parole, ce qui est significatif dans une société où les femmes sont enfermées dans le silence. Et, comme le dit Eleni Varikas à propos des récits d'esclaves, « la reconstitution créatrice de la narration à la première personne qui transforme le bien meuble en sujet parlant [...] révèle des aspects inédits de la domination et de la liberté ${ }^{24} \gg$.

23. « Il n'est plus une volonté du père. Il va devenir sa propre volonté. » (ES, p. 48)

24. E. Varikas, Penser le sexe et le genre, Paris, PUF, coll. « Question d'éthique», 2006, p. 123. Comme d'autres théoriciennes féministes, Varikas reproche à Butler une conception du genre où tout est gouverné par la fonction réitérative et performative du langage. Elle postule la singularité irréductible du processus cognitif, fait de moments discursifs et non discursifs, et la possibilité de configurations totalement inédites. 


\section{Le détour du conte ou la nécessité de la déréalisation}

Ahmed/Zahra est un être profondément subversif: née femme, elle est faite homme, avant de tenter de renouer avec son identité d'origine. Cet épisode transgenre a été inspiré à Ben Jelloun par un fait divers, mais ne donne pas lieu à un récit réaliste. En fait, la transgression est double, par la nature des faits relatés et par l'identité de la narratrice dans La Nuit sacrée: une femme, même âgée, ne peut tout dire « frontalement ». Dans les sociétés arabo-musulmanes, le poids des tabous est tel que la liberté d'agir, de disposer de soi et de s'exprimer librement n'est pas donnée à une femme (ni à un homme sans doute).

Le récit apparaît comme «déréalisé » par le recours au conte et la transgression est relativisée par sa projection dans le registre du merveilleux, ce qui est aussi un moyen d'opérer une restylisation du féminin, et du genre en général. L'audace de la transformation générique d'Ahmed/ Zahra est ainsi contenue par une forme littéraire ancestrale et canonique du monde arabe. En effet, la structure de L'Enfant de sable met en scène toute une série de conteurs, héritiers de la tradition orale, même si le dispositif retenu rappelle davantage les complexités borgésiennes que Les Mille et Une Nuits, tandis que l'espace narratif et discursif se trouve projeté dans un monde perdu et quasi utopique, le Maghreb précolonial. Dans La Nuit sacrée, la multiplication des scènes oniriques éloigne le texte du canon réaliste et déstabilise le lecteur. Le roman se clôt d'ailleurs par une vision de Zahra où elle retrouve le consul, scène en conformité avec l'indécidabilité générale d'un texte tiraillé entre réel et surréel.

Le chapitre de La Nuit sacrée intitulé « le Jardin parfumé » (NS, p. 39-49), inspiré par la poésie d'Abû-I-Alâ, al-Ma'arrî, le Risalat alGhufran ${ }^{25}$, est significatif de cette tendance de l'écriture. Enlevée par un Cheikh, Zahra est conduite dans un lieu utopique, le village des enfants - « une petite république rêvée et vécue par les enfants » (NS, p. 41) -, espace régi par l'oubli et où se réunissent ceux « dont le cœur a souffert et qui ne nourri[ssen]t plus d'illusion sur le genre humain» (NS, p. 42). L'imagerie de ce " jardin parfumé » rappelle bien sûr le Coran et son paradis aux parfums enivrants, aux fruits exquis et aux jeunes filles toujours pures, mais il s'agit ici d'un monde d'enfants, d'avant la sexualité, représentant sans doute pour Zahra la nostalgie d'un temps d'innocence, qu'elle n'a pas connu sous l'identité d'Ahmed. Ce lieu utopique est toutefois synonyme de mort car les enfants y vivent « en dehors du temps » et «à l'abri des vivants » (NS, p. 49). L'amnésie et la dénégation de la sexualité sont mortifères et Zahra doit fuir si elle veut vivre et tenter d'être elle-même. Le registre du conte merveilleux, même s'il a partie liée avec la sexualité - Bruno Bettelheim l'a bien montré -, offre une cryptographie, une série de 
codes symboliques, reconnus et admissibles par une culture, qui permettent à la fois d'idéaliser et d'euphémiser une réalité trop crue.

Tendant au merveilleux, la parole est également sacralisée par les références au livre fondateur de la civilisation arabo-musulmane, le Coran. Le « Journal » d'Ahmed est d'ailleurs explicitement assimilé au livre sacré : «Des phrases ou des versets se lèvent pour dissiper la brume de l'attente. » (ES, p. 107) Quant au manuscrit, sa présentation suit un véritable rituel qui le sacralise et sa lecture consiste en fait en une récitation, ce qui rejoint le sens même du mot «Coran », qui signifie récitation. Contestant les récupérations théologiques, le consul déclare aimer le Coran « comme une poésie superbe » (NS, p. 79), et l'écriture de Ben Jelloun se fait prose poétique. Fatima se retire pour lire les poètes mystiques (ES, p. 104), également présents dans les propos du conteur Amar, qui cite Ibn Arabi et El Hallaj (ES, p. 146-147), deux grands théoriciens et poètes du soufisme, qui font d'ailleurs figure de passeurs entre islam et christianisme ${ }^{26}$. Il est vrai que les variations identitaires d'Ahmed/Zahra ne sont pas sans évoquer « la manière de nos poètes mystiques pour qui l'apparence était le masque le plus pervers de la vérité » (NS, p. 134). Là encore, Ben Jelloun joue sur les attentes culturelles de son lectorat; il englobe le discours du genre et du sexe, dont le caractère prosaïque et potentiellement subversif s'absorbe dans la scansion d'une parole fortement métaphorique, dans la récitation sacralisante d'un texte profondément poétique.

\section{Conclusion}

On peut lire le diptyque de Tahar Ben Jelloun comme une mise en crise de la masculinité à travers l'itinéraire douloureux de l'être double Ahmed/Zahra. La société arabo-musulmane qu'il met en scène se caractérise par le pouvoir absolu du pater familias, consacré par la loi et la coutume et imposé par la coercition économique et la force physique, ce qui fait apparaître les catégories de la masculinité et de la féminité comme stables et immuables. Cependant, loin de proposer une définition essentialiste des genres, la fiction illustre bien plus une approche constructionniste: le genre s'élabore à partir de la répétition d'un certain nombre d'actes, définis par les normes sociales. Comme le dit le texte, Ahmed n'est pas « une erreur de la nature mais un détournement social » (ES, p. 160).

La portée potentiellement subversive du discours de Ben Jelloun sur la masculinité est néanmoins réduite par le procès de l'écriture et l'apparent retour à la norme accompli par son personnage, qui se retrouve assujetti

26. Ibn Arabi a vécu en Andalousie au temps de l'apogée de la civilisation musulmane (1165-1240); El Hallaj a vécu à Bagdad où il a été crucifié pour blasphème en 922. Le soufisme se caractérise d'abord par le dénuement matériel et le refus des biens de ce monde avant de devenir une théosophie. Voir A.-J. Arberry, Le Soufisme: la mystique de l'islam, trad. J. Gouillard, Paris, Le Mail, 1998. 
aux stéréotypes du féminin. La Nuit sacrée semble ratifier l'asymétrie des genres et le système patriarcal tandis que la transgression peut passer pour une exception confirmant la règle de l'ordre symbolique traditionnel. De manière visible, les structures de la fiction euphémisent la mise en crise de la masculinité, d'une part en passant de la structure narrative polyphonique et fragmentaire de L'Enfant de sable à la monophonie de La Nuit sacrée, texte à la construction plus classique, d'autre part en recourant au registre du merveilleux et au fonds culturel traditionnel du conte arabe, qui tendent à métaphoriser les déviances et à déréaliser le substrat idéologique.

L'orientation prise par La Nuit sacrée peut ainsi inviter à interpréter l'œuvre de Ben Jelloun comme un jeu fictionnel sur les identités sexuelles, mais qui se bornerait au constat du patriarcat universel et indépassable. Ben Jelloun recréerait ainsi un Orient littéraire, où prédomine l'oppression du genre féminin par un masculin hégémonique et totalitaire et, ce faisant, jouerait avec l'hypertexte colonial et les stéréotypes occidentaux sur l'Orient éternel et cruel, mais profondément fascinant. Toutefois, la transgression absolue de L'Enfant de sable est loin d'être abolie par le dispositif fictionnel, et elle ouvre la voie aux lectures féministes, à la dénonciation d'une barbarie archaïque, et à la revendication de l'égalité des sexes dans un monde où elle paraît impensable.

Yves Clavaron

Celec, université Jean-Monnet, Saint-Étienne 